\title{
Editorials
}

\section{Single-lung ventilation in pediatrics}

\author{
Mark Levine MBBCH FRCPC, Peter Slinger MD FRCPC
}

W E have yet to identify the ideal method for lung isolation, particularly in children. Recent improvements in technology, the acquisition of surgical expertise and the reported benefits of video-assisted thoracoscopic surgery (VATS) have resulted in an increased use of this technique in pediatric surgery and therefore a greater demand for one-lung ventilation (OLV) in children. ${ }^{1}$ Many other indications for lung isolation in children are well recognized and include: prevention of soiling by blood or pus, tumour resection, lobectomy, tracheo-esophageal fistula repair and repair of coarctation of the aorta. ${ }^{2}$

In this issue Yun and colleagues describe the use of a new 5-French pediatric wire-guided endobronchial blocker (PWEB), positioned coaxially through a conventional endotracheal tube (ETT) for one-lung ventilation in a $45 \mathrm{~kg}, 14$-yr-old patient undergoing repair of coarctation of the aorta. ${ }^{3}$ Previous reports have described the use of a 9-French wired-guided endobronchial blocker (WEB) positioned coaxially through an ETT in adults and alongside conventional ETTs in children. ${ }^{4}$ The large external diameter of the 9-French catheter has precluded its use through an ETT in children. Although the patient described is, by definition, a pediatric patient, he is large enough for placement of a double lumen ETT or 9French WEB therefore rendering lung isolation less challenging than it would be in a younger child. This is, however, the first report of coaxial placement of a 5French PWEB in a child and represents an advance over the bronchial blockers currently available for use in children. For example, the more commonly used Fogarty catheters are usually placed alongside the ETT and may therefore be more difficult to position and stabilize thus increasing the risk of displacement and complete tracheal obstruction. They may also cause airway damage due to the high pressure nature of the balloons. ${ }^{5}$ The PWEB has a low pressure, elliptical shaped balloon and once the position of the PWEB has been confirmed, the guide wire can be removed providing a channel for lung decompression or supplemental oxygen delivery. The WEB also comes with an elegant four-way airway connector that permits separate air-tight access for the anesthesia circuit, the bronchoscope and the blocker. ${ }^{6}$ Furthermore the blocker port incorporates a variable diaphragm which is useful for stabilizing the WEB.

The authors describe the relative ease with which the new 5-French PWEB can be passed coaxially through a conventional ETT. The manufacturer suggests that the smallest ETT through which a PWEB can be passed is a $4.5-\mathrm{mm}$ tube using a neonatal (2 $\mathrm{mm}$ ) bronchoscope to aid placement. We recently used a PWEB, at our institution, in a five-year-old patient with a 5.5-ETT. The blocker was easy to place and use of the airway connector allowed us to maintain ventilation during positioning of the blocker.

Single-lumen endobronchial tubes, double-lumen tubes and bronchial blockers have all been used for the past 50 years for lung isolation. The choice of technique in the individual patient depends on multiple factors which include the age, size and anatomy of the patient, the training of the anesthesiologist, the availability of equipment and the type of surgery. Double-lumen tubes (DLT) have become the gold standard for the provision of lung isolation in adults because they provide the most reliable isolation and they allow continuous access to both lungs. However, double-lumen tubes are designed for normal adult tracheo-bronchial anatomy. They are relatively poorly adaptable in small children or in patients with abnormal upper or lower airways. The increasing frequency of demands for lung isolation in these less-than-straightforward cases has prompted a resurgence of interest in bronchial blockers.

As with double-lumen tubes, when using a bronchial blocker it is important to remember the ABC's of lung isolation:

From the Department of Anaesthesia, University of Toronto, Hospital for Sick Children, Toronto, Ontario, Canada.

Address correspondence to: Dr. Mark Levine, Department of Anaesthesia, The Hospital for Sick Children, 555 University Avenue, Toronto, Ontario M5G 1X8, Canada. Phone: 416-813-7445; Fax: 416-813-7543; E-mail: mark.levine@sickkids.ca 
A) Know the bronchial anatomy. Only by repeated observation of the normal bronchial anatomy is the anesthesiologist able to reliably place an endobronchial tube or blocker following previous surgery or trauma.

B) Use the fibreoptic bronchoscope for initial placement and after repositioning the patient. Blind positioning of endobronchial tubes or blockers is associated with a high incidence of malpositioning ${ }^{7}$ and it may not be defensible if a patient has an intraoperative complication during thoracic surgery and a fibreoptic bronchoscope was available but not used. ${ }^{8}$

C) Look at the chest $x$-ray and computed tomography scan yourself preoperatively. The large majority of tracheo- bronchial distortions that cause problems during placement of endobronchial tubes or blockers can be seen on the preoperative imaging but such concerns are rarely mentioned in the radiologist's report.

The most widely used blocker over the past two decades has been a Fogarty vascular embolectomy catheter. This is a closed-end catheter and is not designed to function as a bronchial blocker. The recent renewed interest in bronchial blockers has led to the development of several new designs and an appreciation of the characteristics of the ideal bronchial blocker which should include:

i) A balloon shape which tends to stabilize it in the bronchus. Like an ETT cuff this probably should be an oval or "rolling-pin" shape and it should have high volume/low-pressure inflation characteristics at the sealing volume of the bronchus.

ii) Be flexible and easy to manipulate into the required mainstem or lobar bronchus.

iii) Have a channel for deflation and suction distal to the blocker.

iv) Be adaptable for use internal and external to a standard ETT.

v) Have a wide variety of sizes for both adult and pediatric patients.

The Univent ${ }^{\circledR}$ tube (Vitaid Ltd., Lewiston, NY, USA) is a single-lumen tube with a blocker enclosed in a separate internal channel. ${ }^{9}$ Many practitioners have found the blocker stiff and occasionally difficult to position. Because of this the manufacturer has redesigned the blocker to make it more flexible. It is generally not necessary (and not easy) to remove the blocker from the Univent ${ }^{\circledR}$ tube. The blocker is long enough that it can be extended to provide lobar blockade in the majority of cases if required. The Univent ${ }^{\circledR}$ tube is available in pediatric sizes $(3.5 \mathrm{~mm}$ and 4.0 $\mathrm{mm})$. However, the external diameter of the these tubes limit their use to children over the age of four to six years. ${ }^{10}$
Tobias described several interesting methods to adapt the adult 9F WEB catheter technique for use in pediatric patients. ${ }^{4}$ In a nine-year-old $38 \mathrm{~kg}$ child he looped the wire guide externally over the distal end of a standard 6.0- mm ETT. The patient was then intubated with the ETT and WEB side-by-side. Following this, a pediatric bronchoscope was passed through the ETT (which was too small to permit both the bronchoscope and WEB simultaneously) and positioned in the desired bronchus. The WEB was then advanced distally off the ETT and the wire-loop guided it along the bronchoscope. One problem with the WEB design is that it is not always easy to position using the provided wire-guide loop system, the blocker can get stuck at the carina. It is important that the blocker and bronchoscope be well lubricated. It can be particularly difficult to reposition the WEB once the wire-guide loop is removed. The search for the ideal blocker is not over.

Several general principles to increase the success rate of lung isolation with blockers are:

i) Position the deflated blocker in the desired bronchus immediately after intubation when the patient is supine, before turning the patient for surgery. It is difficult to manipulate a blocker up into the non-dependent lung after the patient has been placed in the lateral position.

ii) Use a blocker preferentially for left $v$ s right-sided surgery and position the blocker as far distally in the bronchus as possible since blockers tend to migrate proximally. Bauer et al. compared lung isolation with left-sided double- lumen tubes, left bronchial blockers and right bronchial blockers. ${ }^{1}$ Left bronchial blockers took longer to position than DLT's or right-sided blockers. However, lung deflation was satisfactory in all DLT or left blocker cases but in only one-half of the right blocker cases.

iii) Blockers are particularly useful for OLV in nonpulmonary intrathoracic procedures such as esophageal, vascular or vertebral surgery. There is less manipulation of the lung in these cases and less accidental dislodgment of the blocker.

iv) Use a blocker preferentially for open thoracotomies $v s$ thoracoscopies. The lung deflation is slower with a blocker and this tends to delay surgery, particularly in VATS. With an open hemi-thorax the surgeon can retract incompletely deflated lung out of the field but this is not possible during VATS.

v) Use a video camera attached to the fibreoptic bronchoscope, a video monitor and an assistant to position the blocker. This allows the assistant to help by controlling the bronchoscope while you manipulate the blocker. It takes four hands to position a blocker. 
The PWEB may not be the ideal solution for lung isolation but it does have many desirable features for use in pediatric thoracic anesthesia. These include a blocker with small external diameter which is specifically designed for airway use and incorporates a low-pressure elliptical-shaped balloon, a lumen for lung decompression and oxygenation and an airtight airway adapter which allows for efficient positioning while maintaining ventilation and bronchoscopically guided positioning. Further experience with this device is needed to determine where it will fit in the armamentarium of the pediatric thoracic anesthesiologist.

\section{Ventilation unilatérale en pédiatrie}

Nous sommes toujours à la recherche de la méthode idéale d'exclusion pulmonaire, en particulier chez les enfants. Les progrès technologiques récents, l'acquisition de compétences chirurgicales et des articles sur les avantages de la chirurgie thoracoscopique vidéoassistée (CTVA) ont conduit à un usage croissant de cette technique en chirurgie pédiatrique et, par conséquent, à une plus grande demande pour la ventilation unilatérale (VUL) chez les enfants. ${ }^{1}$ De nombreuses autres indications d'exclusion pulmonaire pédiatrique sont bien connues et comprennent : la prévention de contamination par du sang ou du pus, la résection d'une tumeur, la lobectomie, la correction d'une fistule trachéo-œsophagienne ou d'une coarctation de l'aorte. ${ }^{2}$

Dans le présent numéro, Yun et ses collègues décrivent l'utilisation d'un nouveau bloqueur endobronchique pédiatrique avec guide métallique (BEPG) 5-French, passant de façon coaxiale à travers un tube endotrachéal (TET) classique pour la ventilation unilatérale chez un patient de 14 ans, pesant 45 $\mathrm{kg}$, hospitalisé pour la réparation d'une coarctation de l'aorte. ${ }^{3}$ Des articles précédents ont fait mention de l'usage d'un bloqueur endobronchique avec guide métallique (BEG) 9-French passant aussi de façon coaxiale à travers un TET chez des adultes ou le long d'un TET traditionnel chez des enfants. ${ }^{4}$ Étant donné son grand diamètre externe, le cathéter 9-French ne peut être passé dans un TET chez les enfants. Bien que le patient décrit ici soit un enfant, par définition, sa morphologie aurait permis l'usage d'un TET à double lumière ou un BEG 9-French rendant ainsi l'ex- clusion pulmonaire plus facile que chez un enfant plus jeune. L'article de Yun est toutefois le premier à rapporter la mise en place coaxiale d'un BEPG 5-French chez un enfant. C'est donc un progrès par rapport aux bloqueurs bronchiques utilisés en pédiatrie. Par exemple, les cathéters Fogarty les plus utilisés sont habituellement insérés le long du TET et peuvent donc être plus difficiles à placer et à stabiliser. Cette situation augmente le risque de déplacement et d'obstruction trachéale complète. Ces cathéters peuvent aussi causer des lésions aux voies respiratoires étant donné la caractéristique de haute pression des ballonnets. ${ }^{5}$ Le BEPG comporte un ballonnet de basse pression, de forme elliptique et, une fois que sa bonne position est confirmée, le guide métallique peut être retiré laissant un canal qui permet la décompression pulmonaire ou l'apport d'oxygène supplémentaire. Le BEG se présente aussi muni d'un élégant raccord à quatre voies qui permet $d$ 'isoler un accès hermétique pour le circuit d'anesthésie, le bronchoscope et le bloqueur. ${ }^{6}$ De plus, la fenêtre du bloqueur comprend un diaphragme variable, utile pour stabiliser le BEG.

Les auteurs décrivent la facilité relative avec laquelle le nouveau BEPG 5-French peut être inséré de façon coaxiale à travers un TET traditionnel. Le fabricant propose, comme le plus petit TET au travers duquel un BEPG puisse être inséré, un tube de 4,5 $\mathrm{mm}$ et l'utilisation d'un bronchoscope néonatal de 2 $\mathrm{mm}$ pour faciliter la mise en place. Nous avons récemment utilisé un BEPG avec un TET 5,5 chez un enfant de cinq ans. Le bloqueur a été facilement mis en place et l'utilisation du raccord de ventilation nous a permis de maintenir la ventilation pendant l'insertion du bloqueur.

Au cours des 50 dernières années, les tubes endobronchiques à lumière simple ou double et les bloqueurs bronchiques ont tous servi à l'exclusion pulmonaire. Le choix d'une technique dépend de multiples facteurs qui comprennent l'âge, la taille et la morphologie du patient, la formation de l'anesthésiologiste, la disponibilité du matériel et le type de chirurgie. Les tubes à double lumière (TDL) sont devenus la référence en matière d'exclusion pulmonaire chez les adultes parce qu'ils assurent l'exlusion la plus fiable et permettent un accès continu aux deux poumons. Ces tubes sont cependant conçus pour l'anatomie trachéo-bronchique adulte normale. Ils sont relativement peu adaptables chez les petits enfants ou chez les patients dont les voies aériennes supérieures ou inférieures sont anormales. Les exigences croissantes reliées à l'exclusion pulmonaire de cas assez complexes ont ravivé l'intérêt pour les bloqueurs bronchiques. 
Que l'on utilise un bloqueur bronchique ou un tube à double lumière, il est important de se rappeler l'ABC de l'exclusion pulmonaire :

A) Connaître l'anatomie bronchique. Seule l'observation répétée de l'anatomie bronchique normale permet à l'anesthésiologiste de placer un tube endobronchique ou un bloqueur en toute sécurité à la suite d'une intervention chirurgicale ou d'un traumatisme.

B) Utiliser un fibroscope bronchique pour la mise en place initiale et à la suite d'un changement de position du patient. L'insertion à l'aveugle des tubes endobronchiques ou des bloqueurs entraîne une forte incidence d'erreurs de positions ${ }^{7}$ qui peuvent être injustifiables en présence de complications peropératoires pendant l'intervention chirurgicale thoracique alors qu'il y a sur place un fibroscope bronchique non utilisé. $^{8}$

C)Vérifier soi-même les radiographies des poumons et les résultats de la tomodensitométrie avant l'opération. La grande majorité des distorsions trachéo-bronchiques qui compliquent la mise en place de tubes endobronchiques ou de bloqueurs peuvent être visualisées avant l'opération sur les épreuves d'imagerie mais elles sont rarement mentionnées dans le rapport du radiologiste.

Le bloqueur le plus utilisé au cours des deux dernières décennies a été le cathéter pour embolectomie vasculaire Fogarty. C'est un cathéter borgne, non conçu comme un bloqueur bronchique. Le regain d'intérêt pour les bloqueurs bronchiques a mené à la réalisation de nouveaux modèles. Les caractéristiques d'un bloqueur bronchique idéal devraient comprendre :

i) Une forme de ballonnet qui tend à le stabiliser à l'intérieur de la bronche. Il devrait être ovale comme dans le cas d'un TET à ballonnet ou avoir la forme d'un rouleau et posséder les caractéristiques de grand volume/basse pression quand il est gonflé de manière à assurer l'étanchéité de la bronche.

ii) La flexibilité et la facilité de manipulation à l'intérieur de la bronche principale ou lobaire.

iii) Un canal permettant le dégonflement et l'aspiration distale au bloqueur.

iv) L'adaptabilité à un TET régulier pour un usage interne et externe.

v) Une grande diversité de tailles autant pour les adultes que pour les enfants.

Le tube Univent ${ }^{\circledR}$ (Vitaid Ltd., Lewiston, NY, USA) est un tube monolumière comprenant un bloqueur dans un canal interne séparé. ${ }^{9}$ De nombreux médecins trouvent le bloqueur rigide et parfois difficile à installer. En conséquence, le fabricant a redes- siné le bloqueur pour le rendre plus flexible. Il n'est généralement pas nécessaire (et pas facile) de retirer le bloqueur du tube Univent ${ }^{\circledR}$. Le bloqueur peut être allongé pour fournir un blocage lobaire dans la majorité des cas si nécessaire. Le tube Univent ${ }^{\circledR}$ existe en différentes tailles pour les enfants $(3,5 \mathrm{~mm}$ et 4,0 $\mathrm{mm})$. Cependant, le diamètre externe de ces tubes limite leur utilisation aux enfants de plus de quatre à six ans. ${ }^{10}$

Tobias a présenté quelques méthodes intéressantes pour adapter la technique du cathéter BEG 9F adulte aux patients pédiatriques. ${ }^{4}$ Dans le cas d'un enfant de neuf ans, de $38 \mathrm{~kg}$, il a enroulé le guide métallique à la partie distale externe d'un TET standard de 6,0 $\mathrm{mm}$. Le patient a ensuite été intubé avec le TET et le BEG côte à côte. Par la suite, un bronchoscope pédiatrique a été introduit à travers le TET (lequel était trop petit pour permettre d'introduire le bronchoscope et le BEG simultanément) et mis en place dans la bronche choisie. Le BEG a été ensuite poussé en aval du TET et guidé par la boucle métallique le long du bronchoscope. L'un des problèmes engendrés par la forme du BEG est qu'il n'est pas toujours facile à installer en utilisant le système en boucle du guide métallique fourni, le dispositif ayant tendance à bloquer à la carène. C'est important que le bloqueur et le bronchoscope soient bien lubrifiés. Il peut être particulièrement difficile de replacer le BEG une fois que la boucle du guide métallique est retirée. Le bloqueur idéal est encore à découvrir.

Certains principes généraux s'appliquent pour augmenter le taux de succès de l'exclusion pulmonaire avec les bloqueurs :

i) Placer le bloqueur non gonflé dans la bronche choisie immédiatement après l'intubation, le patient étant en décubitus dorsal, avant de retourner le patient pour l'intervention chirurgicale. Il est difficile de manipuler un bloqueur vers le haut dans le poumon non déclive après que le patient a été installé en position latérale.

ii) Utiliser de préférence un bloqueur pour intervention chirurgicale gauche, par opposition à droite, et placer le bloqueur aussi loin que possible en aval dans la bronche, car les bloqueurs tendent à revenir en amont. Bauer et coll. ont comparé l'exclusion pulmonaire réalisée avec des tubes à double lumière gauches, des bloqueurs bronchiques gauches et droits. ${ }^{1}$ Les bloqueurs bronchiques gauches sont plus longs à installer que les TDL ou que les bloqueurs droits. Cependant, l'affaissement du poumon a été satisfaisant dans tous les cas d'utilisation de TDL ou de bloqueur gauche, mais dans la moitié seulement des cas d'utilisation de bloqueur droit. 
iii) Les bloqueurs sont particulièrement utiles pour la VUL pendant les interventions intrathoraciques non pulmonaires comme les opérations œsophagiennes, vasculaires ou vertébrales. Dans ces cas, il y a moins de manipulation du poumon et moins de déplacement accidentel du bloqueur.

iv) Utiliser de préférence un bloqueur pour thoracotomies ouvertes $v s$ thoracoscopies. L'affaissement du poumon est plus lent avec un bloqueur, ce qui tend à retarder l'opération, surtout en CTVA. En présence d'un hémi-thorax ouvert, le chirurgien peut déplacer hors champ un poumon partiellement affaissé, mais cette situation n'est pas possible pendant une CTVA.

v) Utiliser une caméra vidéo raccordée au fibroscope bronchique, un moniteur vidéo et avoir un assistant pour mettre en place le bloqueur. L'assistant peut ainsi aider en contrôlant le bronchoscope pendant que vous manipulez le bloqueur. Il faut quatre mains pour installer un bloqueur.

Le BEPG n'est peut-être pas la solution idéale à l'exclusion pulmonaire, mais il présente de nombreuses caractéristiques recherchées pour l'usage anesthésique pendant la chirurgie thoracique pédiatrique. Ce qui comprend un bloqueur au petit diamètre externe conçu spécialement pour l'intubation et comporte un ballonnet de basse pression et de forme elliptique, un canal pour la décompression pulmonaire et l'oxygénation et un adaptateur d'intubation étanche qui permet une mise en place efficace tout en maintenant la ventilation et l'installation guidée par le bronchoscope. Nous avons besoin d'expérimenter plus avant cet outil afin de découvrir comment il s'intègre dans l'arsenal thérapeutique de l'anesthésiologiste en chirurgie pédiatrique thoracique.

References

1 Milanez de Campos JR, Filho LOA, Werebe EC, et al. Thoracoscopy in children and adolescents. Chest 1997; 111: 494-7.

2 Haynes SR, Bonner S. Review article: anaesthesia for thoracic surgery in children. Paediatr Anaesth 2000; 10: 237-51.

3 Yun ES, Saulys A, Popic PM, Arndt GA. Single-lung ventilation in a pediatric patient using a pediatric fibreoptically-directed wire-guided endobronchial blocker. Can J Anesth 2002; 49: 256-61.

4 Tobias JD. Variations on one-lung ventilation. J Clin Anesth 2001; 13: 35-9.

5 Borchardt RA, LaQuaglia MP, McDowall RH, Wilson

$R S$. Bronchial injury during lung isolation in a pediatric patient. Anesth Analg 1998; 87: 324-5.

6 Arndt GA, Buchika S, Kranner PW, DeLessio ST. Wireguided endobronchial blockade in a patient with a lim- ited mouth ospening. Can J Anesth 1999; 46: 87-9.

7 Klein U, Karzai W, Bloos F, et al. Role of fiberoptic bronchoscopy in conjunction with the use of doublelumen tubes for thoracic anesthesia: a prospective study. Anesthesiology 1998; 88: 346-50.

8 Pennefather SH, Russell GN. Placement of double lumen tubes-time to shed light on an old problem. $\mathrm{Br}$ J Anaesth 2000; 84: 308-10.

9 Slinger P. Con: the Univent tube is not the best method of providing one-lung ventilation. J Cardiothorac Vasc Anesth 1993; 7: 108-12.

10 Tobias JD. Anaesthetic implications of thoracoscopic surgery in children. Paediatr Anaesth 1999; 9: 103-10.

11 Bauer C, Winter C, Hentz JG, Ducrocq X, Steib A, Dupeyron JP. Bronchial blocker compared to doublelumen tube for one-lung ventilation during thoracoscopy. Acta Anaesthesiol Scand 2001; 45: 250-4. 